\title{
Linear and Nonlinear Diffraction of Dipolar Spin Waves in Yttrium Iron Gar- net Films Observed by Space- and Time-Resolved Brillouin Light Scattering
}

\author{
O. Büttner, M. Bauer, S.O. Demokritov, and B. Hillebrands \\ Fachbereich Physik and Zentrum für Lasermeßtechnik und Diagnostik, Universität Kaiserslautern, 67663 Kaiserslautern, Germany
}

\author{
Yu.S. Kivshar \\ Optical Sciences Centre, Australian National University, Canberra ACT 0200, Australia
}

V. Grimalsky and Yu. Rapoport

T. Shevchenko Kiev State University, 252601, Kiev, Ukraine

\author{
A.N. Slavin \\ Department of Physics, Oakland University, Rochester, Michigan 48309, USA
}

\begin{abstract}
A new advanced space- and time-resolved Brillouin light scattering (BLS) technique is used to study diffraction of two-dimensional beams and pulses of dipolar spin waves excited by strip-line antennas in tangentially magnetized garnet films. The new technique is an effective tool for investigations of two-dimensional spin wave propagation with high spatial and temporal resolution. Linear effects, such as the unidirectional excitation of magnetostatic surface waves and the propagation of backward volume magnetostatic waves (BVMSW) in two preferential directions due to the non-collinearity of their phase and group velocities are investigated in detail. In the nonlinear regime stationary and non-stationary self-focusing effects are studied. It is shown, that non-linear diffraction of a stationary BVMSW beam, having a finite transverse aperture, leads to self-focusing of the beam at one spatial point. Diffraction of a finite-duration (non-stationary) BVMSW pulse leads to space-time self-focusing and formation of a strongly localized two-dimensional wave packet (spin wave bullet). Numerical modeling of the diffraction process by using a variational approach and direct numerical integration of the two-dimensional non-linear Schrödinger equation provides a good qualitative description of the observed phenomena.
\end{abstract}

\section{Introduction}

Magnetostatic spin waves in yttrium-iron garnet (YIG) films provide a superb testing ground to study linear and nonlinear wave processes in dispersive, diffractive, and anisotropic media with relatively low dissipation $[1,2]$. Nonlinear properties of magnetostatic waves in YIG films are therefore anisotropic in the film plane as well. The threshold of nonlinearity determined by the dissipation parameter is so low that a wide variety of nonlinear wave effects, like formation of envelope solitons [3], modulational [4], decay and kinetic [5] instabilities, can be observed for input microwave powers below $1 \mathrm{~W}$.

In films the wave process is easily accessible from the surface. Inductive probes [6, 7], thermo-optical methods [8], and Faraday rotation measurements [9] were used to study magnetostatic wave processes in YIG films. It is, however, the recently developed method of space- and time-resolved Brillouin light scattering (BLS) [10, 11, 12], which provided a major leap in this field due to its high resolution, sensitivity, dynamic range and stability. Using this method it is now possible not only to reproduce all the results obtained previously for stationary wave processes, but also to investigate non-stationary nonlinear wave processes like propagation of intensive wave packets of finite duration and finite transverse aperture (pulse-beams) that are simultaneously affected by dispersion, diffraction, nonlinearity and dissipation [13].

In this paper we present the results of our investigations of linear and nonlinear diffraction of dipolar spin waves propagating in tangentially magnetized YIG films. We first dem- onstrate the excellent applicability of our method to the observation of well-known linear properties of magnetostatic waves like non-reciprocity of magnetostatic surface wave (MSSW) and reciprocity of backward volume magnetostatic wave (BVMSW) excitations. We discuss non-collinearity of the phase and the group velocity for waves propagating at an arbitrary angle to the in-plane bias magnetic field, and we show the existence of two preferential directions of the wave propagation for the wave beams radiated in a wide angle in the transverse direction. Next we show that our method allows to observe and investigate in detail new nonlinear effects such as the stationary one-dimensional self-focusing effect of microwave excited BVMSW beams leading to the formation of spatial spin wave solitons, and the nonstationary spatio-temporal self-focusing effect of twodimensional propagating wave packets leading to the formation of highly localized quasi-stable wave pulses - spin wave bullets. The last effect was predicted in optics [14], but the first experimental observation of this effect has been reported for the system of magnetostatic spin waves propagating in a YIG film [13].

\section{Theoretical background}

The dispersion equation for purely dipolar (non-exchange) magnetostatic waves propagating in a tangentially magnetized ferromagnetic slab of a finite thickness $L$ was first derived by Damon and Eshbach in 1961 [15]. The explicit dispersion equation in the form $\omega=f(\mathrm{k})$, where $\omega$ is the wave frequency and $\mathrm{k}$ is the wavevector, was obtained in [15] only for the case of magnetostatic surface waves (MSSW) propa- 
gating perpendicularly to the direction of the bias magnetic field (see Eq. (23) in [15]). For other directions of wave propagation the dispersion equation has an implicit form. Therefore, for the spectral calculations in this paper we use the approximate explicit dispersion equation obtained in [16] for the lowest (homogeneous) thickness mode $(n=0)$ neglecting the exchange interaction (see Eq. (45) in [16]).

In the following we assume a Cartesian coordinate system oriented such that the film normal is along the $x$-axis, and the external field is aligned along the $z$-axis. In the longwavelength limit ( $k L<1 / 2$ where $L$ is the film thickness) the dispersion equation obtained from Eq. (45) in [16] gives reasonably accurate results for all directions of the in-plane wavevector $\mathrm{k}$, and it has the form

$$
\omega_{0 \mathrm{k}}^{2}(\mathbf{k})=\omega_{\mathrm{H}}\left[\omega_{\mathrm{H}}+\omega_{\mathrm{M}} \mathrm{F}\left(\mathrm{k}_{\mathrm{y}}, \mathrm{k}_{\mathrm{z}}\right)\right]
$$

where the in-plane wavevector $\mathbf{k}=\mathrm{k}_{\mathrm{y}} \mathbf{y}_{\mathbf{0}}+\mathrm{k}_{\mathrm{z}} \mathbf{z}_{\mathbf{0}}$ $\left(\mathrm{k}=\sqrt{\mathrm{k}_{\mathrm{y}}^{2}+\mathrm{k}_{\mathrm{z}}^{2}}\right)$ forms the angle of propagation

$$
\varphi=\arctan \left(\frac{\mathrm{k}_{\mathrm{y}}}{\mathrm{k}_{\mathrm{z}}}\right)
$$

with the direction of the in-plane bias magnetic field $\mathrm{H}=\mathrm{Hz}_{0}$. The external field and the magnetization are expressed in units of frequency: $\omega_{\mathrm{H}}=\gamma \mathrm{H}, \omega_{\mathrm{M}}=\gamma 4 \pi \mathrm{M}_{\mathrm{z}} \approx \gamma 4 \pi \mathrm{M}_{0}$, with $M_{z}$ the magnitude of the $z$-component of the static magnetization, and $M_{o}$ the saturation magnetization in the medium when no waves are excited. $\gamma$ is the magnitude of the gyromagnetic ratio for the electron spin $(\gamma / 2 \pi=2.8 \mathrm{MHz} / \mathrm{Oe})$. The matrix element $F$ describes the dipole-dipole interaction in Eq. (1). It is determined by the expression [16]

$$
\mathrm{F}\left(\mathrm{k}_{\mathrm{y}}, \mathrm{k}_{\mathrm{z}}\right)=1+\mathrm{P}(\mathrm{k})[1-\mathrm{P}(\mathrm{k})]\left(\frac{\omega_{\mathrm{M}}}{\omega_{\mathrm{H}}}\right)\left(\frac{\mathrm{k}_{\mathrm{y}}^{2}}{\mathrm{k}^{2}}\right)-\mathrm{P}(\mathrm{k})\left(\frac{\mathrm{k}_{\mathrm{z}}^{2}}{\mathrm{k}^{2}}\right)
$$

with

$$
\mathrm{P}(\mathrm{k})=1-\frac{1-\exp (-\mathrm{kL})}{\mathrm{kL}} .
$$

It is clear from Eqs. (1)-(4), that the spectrum of dipolar spin waves in a tangentially magnetized film is anisotropic, and the dispersion properties of waves depend significantly on

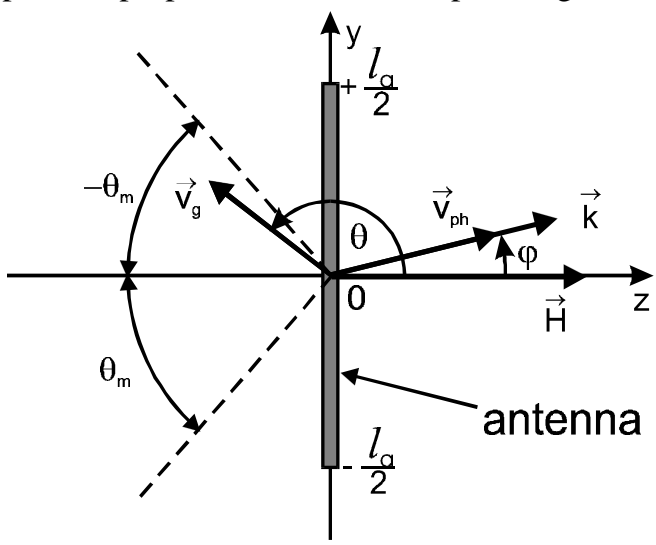

Fig. 1: Geometry of the excitation of a backward volume magnetostatic wave (BVMSW) by a microwave antenna. The dashed bar in the middle shows the position of the antenna of the length $l_{\mathrm{a}}$, extended along the ydirection. The bias magnetic field is parallel to the positive $z$-direction. The angle $\varphi$ indicates the direction of the wavevector (or phase velocity) of the radiated wave relative to the direction of the bias field $H$. The angle $\theta$ indicates the direction of the group velocity $v_{\mathrm{g}}$. Dashed lines show the two preferential directions $\left( \pm \theta_{\mathrm{m}}\right)$ of the group velocity in a wide wave beam radiated by the antenna (see also Fig. 2). the angle of propagation, $\varphi$. In such an anisotropic spectrum the direction of the wave phase velocity $\mathbf{v}_{\mathrm{ph}}=\omega(\mathbf{k}) \mathbf{k} / \mathrm{k}^{2}$, which is collinear with the direction of the wavevector $\mathrm{k}$, does not, in general, coincide with the direction of the group velocity $\mathbf{v}_{\mathrm{g}}=\partial \omega(\mathbf{k}) / \partial \mathbf{k}$ of a wave packet. The angle $\theta$ between the direction of $\mathrm{v}_{\mathrm{g}}$ and the bias field $\mathrm{H}$ is determined by the expression

$$
\theta=\arctan \left(\frac{\mathrm{v}_{\mathrm{gy}}}{\mathrm{v}_{\mathrm{gz}}}\right)=\arctan \left(\frac{\partial \omega / \partial \mathrm{k}_{\mathrm{y}}}{\partial \omega / \partial \mathrm{k}_{\mathrm{z}}}\right) .
$$

The excitation of dipolar spin waves in a magnetic film is usually made by a microstrip antenna. The direction of the wavevector is chosen by the orientation of the antenna, while the initial in-plane width of the wave beam is determined by the antenna aperture, $l_{a}$, and the carrier wavevector $k_{0 z}$. The typical geometry of excitation of a BVMSW wave packet is shown in Fig. 1. The transverse angular width of the excited wave packet, characterized by the maximum angle of propagation, $\varphi_{\max }$, is determined by the antenna aperture $l_{a}$ :

$$
\varphi_{\max }=\arctan \left(\frac{\mathrm{k}_{\mathrm{y}}^{\max }}{\mathrm{k}_{0 \mathrm{z}}}\right) \approx \arctan \left(\frac{2 \pi}{\mathrm{k}_{0 \mathrm{z}} \mathrm{l}_{\mathrm{a}}}\right) .
$$

with $k_{y}^{\text {max }}$ the maximum transverse wavevector. The value of the carrier BVMSW wavevector $k_{0 z}$ for a given excitation frequency $\omega_{0}$ is determined from the dispersion equation Eq. (1).

When the wave beam is excited by the antenna in a sufficiently narrow angle in the transverse (y) direction, it propagates perpendicular to the antenna aperture (in this case along the $z$-direction which is parallel to the bias magnetic field $\mathrm{H}$ ). Using Eqs. (1-5) for given values of the excitation frequency $\omega=\omega_{0}$ (determined by the frequency of the external microwave signal, the bias magnetic field $\mathrm{H}$, and the saturation magnetization of the material $\mathrm{M}_{0}$ ), it is easy to calculate the dependence of the direction of the group velocity on the direction of the phase velocity in the excited wave beam $\theta=f(\varphi)$. This dependence, calculated for the parameters of our experiment described below, is presented in Fig. 2. It is clear, that for a sufficiently wide initial wave packet $\left(\mathrm{k}_{\mathrm{y}}^{\max } / \mathrm{k}_{0 \mathrm{z}} \approx 1, \varphi>35^{\circ}\right)$, excited by a short antenna or a point-like source, there are two preferential directions of the group velocity $\theta=\pi \pm \theta_{\mathrm{m}}$, which do not coincide with the direction normal to the antenna. These directions are shown in Fig. 1 by broken lines.

This linear diffraction effect, typical for anisotropic media, has been studied in early diffraction experiments performed in YIG films using inductive probes [7]. Upon increasing the carrier wavevector $k_{0 z}$ or/and the antenna aperture $l_{a}$, the initial angular width $\varphi_{\max }$ of the excited wave beam decreases, and the excited beam becomes narrower in the transverse direction. For such a narrow beam the dependence $\theta=f(\varphi)$ is almost linear (see the central part in Fig. 2), and the wave beam energy is radiated mainly perpendicular to the antenna aperture.

So far only stationary beams of dipolar spin waves excited by a continuous external microwave power were discussed. If the excitation is made by a microwave pulse of a finite duration $T$, the excited wave packet has also a finite length 
along the direction of propagation $l_{\mathrm{z}}=v_{\mathrm{gz}} T$. Thus, the excited wave packet is two-dimensional, and has a finite spectral width in k-space along both in-plane directions:

$$
\Delta \mathrm{k}_{\mathrm{y}} \approx \mathrm{k}_{\mathrm{y}}^{\max } \approx \frac{2 \pi}{1_{\mathrm{a}}}, \Delta \mathrm{k}_{\mathrm{z}} \approx \frac{2 \pi}{\mathrm{v}_{\mathrm{gz}} \mathrm{T}} .
$$

It is well-known, that, due to the strong exchange interaction between spin magnetic moments in a ferromagnet, the total length of the magnetization vector $M$ is conserved (see, e.g., [17]). Thus, when the variable magnetization $\mathrm{m}$ in the plane perpendicular to the $z$-axis increases, the magnitude of the constant magnetization along the $z$-axis is reduced, and which yields a value for the $z$-component of the magnetization of $M_{z}=M_{0} \sqrt{1-\left(m / M_{0}\right)^{2}}$. The nonlinearity (i.e., the dependence of the wave frequency on the wave amplitude) can be introduced in the dispersion law Eq. (1) through the parameter $\omega_{M}=4 \pi \gamma M_{z}$, since it depends on the value of the variable magnetization, $m$. By expanding the square root we obtain $\quad \mathrm{M}_{\mathrm{z}}=\mathrm{M}_{0}\left(1-\frac{1}{2}\left(\mathrm{~m} / \mathrm{M}_{0}\right)^{2}\right)=\mathrm{M}_{0}\left(1-|\psi|^{2}\right) \quad$ with $\psi^{2}=\mathrm{m}^{2} / 2 \mathrm{M}_{0}^{2}$ the dimensionless amplitude of the variable magnetization in the excited microwave spin-wave packet (see [18] and Chapter 9 in [1]).

When the excited wave packet is spectrally narrow along the both in-plane directions $\left(\Delta \mathrm{k}_{\mathrm{y}}, \Delta \mathrm{k}_{\mathrm{z}}<<\mathrm{k}_{0 \mathrm{z}}\right)$, and the amplitude of the wave packet is small $\left(|\psi|^{2}<<1\right)$, it is possible to expand the dispersion equation (1) in a Taylor series near the working point $\left(\omega=\omega_{0}, \mathrm{k}=k_{0 z} \mathrm{z}_{0},|\psi|^{2}=0\right)$ :

$$
\begin{aligned}
\omega\left(\mathbf{k},|\psi|^{2}\right)= & \omega_{0}\left(\mathrm{k}_{0 \mathrm{z}}\right)+\frac{\partial \omega}{\partial \mathrm{k}_{\mathrm{z}}} \Delta \mathrm{k}_{\mathrm{z}}+\frac{1}{2} \frac{\partial^{2} \omega}{\partial \mathrm{k}_{\mathrm{z}}^{2}}\left(\Delta \mathrm{k}_{\mathrm{z}}\right)^{2} \\
& +\frac{1}{2} \frac{\partial^{2} \omega}{\partial \mathrm{k}_{\mathrm{y}}^{2}}\left(\Delta \mathrm{k}_{\mathrm{y}}\right)^{2}+\frac{\partial \omega}{\partial|\psi|^{2}}|\psi|^{2}+\ldots
\end{aligned}
$$

Using the "method of envelopes" developed in [19], it is possible to obtain from the expansion Eq. (8) an equation, which describes the space and time evolution of the slowly varying envelope function $U(y, z, t)$ of the excited wave packet: $\mathrm{U}(\mathrm{y}, \mathrm{z}, \mathrm{t})=\psi(\mathrm{y}, \mathrm{z}, \mathrm{t}) \cdot \exp \left[\mathrm{i}\left(\omega_{0} \mathrm{t}-\mathrm{k}_{0 \mathrm{z}} \mathrm{z}\right)\right]$. We obtain the so-called (2+1)-dimensional Nonlinear Schrödinger

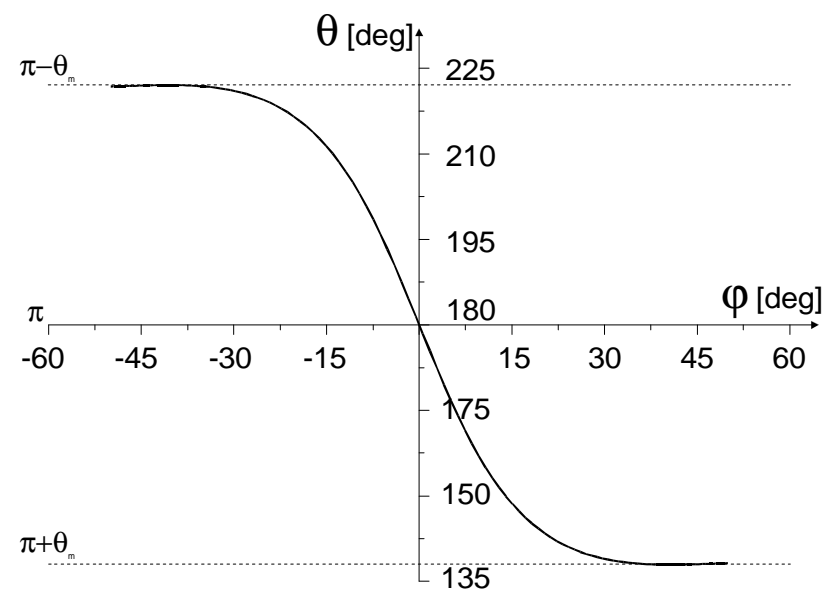

Fig. 2: Dependence of the direction of the group velocity $\theta$ on the direction of the phase velocity $\varphi$ in a beam of magnetostatic waves excited in a tangentially magnetized BIG (Bi substituted YIG) film (film thickness $\mathrm{L}=1.5 \mu \mathrm{m}$, saturation magnetization $4 \pi \mathrm{M}_{\mathrm{s}}=1750 \mathrm{Oe}$, bias magnetic field $\mathrm{H}=2298 \mathrm{Oe})$. The value of $\theta_{\mathrm{m}}$ in this case is $42^{\circ}$. equation (NSE) (see e.g. [18]) :

$$
\mathrm{i}\left(\frac{\partial \mathrm{U}}{\partial \mathrm{t}}+\mathrm{v}_{\mathrm{g}} \frac{\partial \mathrm{U}}{\partial \mathrm{z}}\right)+\frac{1}{2} \mathrm{D} \frac{\partial^{2} \mathrm{U}}{\partial \mathrm{z}^{2}}+\mathrm{S} \frac{\partial^{2} \mathrm{U}}{\partial \mathrm{y}^{2}}-\mathrm{N}|\mathrm{U}|^{2} \mathrm{U}=-\mathrm{i} \omega_{\mathrm{r}} \mathrm{U},
$$

where $\quad \mathrm{v}_{\mathrm{g}}=\partial \omega /\left.\partial \mathrm{k}_{\mathrm{z}}\right|_{\mathrm{k}_{02}}$ is the group velocity,

$\mathrm{D}=\partial^{2} \omega /\left.\partial \mathrm{k}_{\mathrm{z}}^{2}\right|_{\mathrm{k}_{0 \mathrm{z}}}$ and $\mathrm{S}=\partial \omega /\left.\partial \mathrm{k}_{\mathrm{y}}^{2}\right|_{\mathrm{k}_{0 \mathrm{z}}}$ are the dispersion and diffraction coefficients, $\mathrm{N}=\partial \omega /\left.\partial|\mathrm{U}|^{2}\right|_{\mathrm{k}_{0 z}}$ is the nonlinear coefficient, and $\omega_{\mathrm{r}}=\gamma \Delta \mathrm{H}$ is the dissipation parameter proportional to the ferromagnetic resonance (FMR) halflinewidth $\Delta H$. The weak dissipation contribution, which is nevertheless important in YIG films [20], has been introduced in Eq. (9) phenomenologically. The coefficients $v_{g} D$, $S$ and $N$ in Eq. (9) have been calculated for different directions of spin wave propagation in Chapter 9 of [1]. A more rigorous calculation of the nonlinear coefficient $N$ is reported in [21] using a classical Hamiltonian formalism for spin waves in magnetic films. The $(2+1)$-dimensional nonlinear Schrödinger equation (two in-plane coordinates plus time) with dissipation, Eq. (9), will be our main model used to describe the observed nonlinear diffraction effects for dipolar spin waves in this paper.

\subsection{Modulational instabilities}

The most interesting case to study two-dimensional nonlinear spin wave processes is a case of a tangentially magnetized YIG film. Here the properties of waves propagating in different in-plane directions are dramatically different.

For backward-volume magnetostatic waves (BVMSW), propagating along the direction of the bias magnetic field, the nonlinear coefficient $N$ is negative, while the coefficients $D$ and $S$ describing dispersion and diffraction are both positive (see Chapter 9 in [1]). Thus the Lighthill criterion [22] for modulational instability is fulfilled in both in-plane directions $(S N<0, D N<0)$ and the BVMSW are susceptible to both self-modulation in the direction of propagation $(z)$ and to self-focusing in the transverse direction $(y)$.

In the case of a sinusoidal input signal supplied to a relatively short input antenna situated on a wide YIG film sample the excited dipolar spin waves are monochromatic, and they propagate in a relatively wide angle in the transverse $(y)$ direction. These monochromatic waves are not affected by dispersion, but due to the presence of different directions of wavevectors in the excited beam they are strongly affected by diffraction. Due to the competition between diffraction and nonlinearity, transverse modulational instability develops, and this leads to stationary self-focusing of the wave beam and to the formation of spatial spin wave envelope solitons $[13,23]$. This effect can be described by a onedimensional reduction of the NSE Eq. (9) with $\partial \mathrm{U} / \partial \mathrm{t}=0$ and $D=0$.

In the opposite case, when a pulsed input signal propagates in a narrow strip of a YIG film (spin-wave waveguide), where the strip width is smaller than the wavelength of the transverse modulational instability, the signal is not affected by diffraction, but, due to the presence of many different spectral components in the pulse, the signal is strongly affected by dispersion. The competition between dispersion 
and nonlinearity leads to a longitudinal self-modulation of the signal and to the formation of temporal spin wave envelope solitons [24]. Formation of temporal envelope solitons can also be described by a one-dimensional reduction of the NSE Eq. (9); only in this case we should assume that $S=0$.

Both these effects can be observed simultaneously if a pulsed input signal excites a two-dimensional wave packet of BVMSW modes in a wide YIG film sample. In this case modulational instability takes place in both in-plane directions and leads to a spatio-temporal self-focusing of the wave packet and to initial stages of the wave collapse, when the whole energy of the packet is concentrated in a small spatial region [12]. The full (2+1)-dimensional NSE is necessary for the theoretical description of this effect.

The behavior of magnetostatic surface waves (MSSW), propagating perpendicular to the direction of the bias magnetic field, is very different. MSSW modes are modulationally stable and can only form dark spin wave solitons [25]. These waves, however, demonstrate non-reciprocal properties, and a microstrip antenna effectively radiates these waves in only one direction [26].

It is worth noting, that dissipation in YIG films, although weak, plays an important qualitative role in all the nonlinear spin wave processes. It determines the power threshold $|\mathrm{U}|_{\text {th }}^{2}$ of temporal soliton formation and spatial self-focusing [20, 27]:

$$
|\mathrm{U}|_{\mathrm{th}}^{2}=\frac{\omega_{\mathrm{r}}}{|\mathrm{N}|}
$$

and it limits the catastrophic spatio-temporal self-focusing and collapse of two-dimensional non-stationary wave packets of BVMSW modes [12], see below.

Most of the experiments on nonlinear spin waves in YIG films reported in the literature were made by means of microwave spectroscopy. In these experiments spin waves in the $\mathrm{GHz}$ frequency range are excited by a microstrip antenna. The propagating spin waves are detected by a second microstrip antenna that is connected to a microwave detector [1, 2]. By construction, the receiving antenna integrates the spin wave intensity along its length, and, therefore, all the information about the transverse distribution of the intensity in the propagating spin wave packet is lost. Thus, the processes of formation and propagation of temporal spin wave envelope solitons in all these studies were treated in the past as essentially being one-dimensional.

\section{The space- and time-resolved Brillouin light scattering technique}

The first attempts to study the two-dimensional character of linear and nonlinear spin wave processes in YIG films were made by using the method of inductive probe scanning, where a small wire loop was used to scan the sample and to measure the magnetic field of the spin waves [6]. In other experiments the Faraday rotation or a thermal method that measures the heating of the sample caused by the waves were used to measure the spin wave intensity [8]. All these methods provide a reasonable spatial resolution in measurements of spin wave intensity, but they do not provide temporal resolution, and, therefore, they are not suitable to study fast non-stationary spin wave processes.
To investigate both the spatial and the temporal properties of spin wave packets, we have developed a new experimental technique based on a standard Brillouin light scattering (BLS) setup. It is schematically shown in Fig. 3. Spin waves are generated by a microwave input antenna. If microwaves with a frequency $\omega_{0}$ are applied to the input antenna, a spin wave is launched with a wavevector determined by the dispersion relation $\omega_{0}(\mathrm{k})$ of the spin wave. The spatial distribution is now measured by scanning the laser beam across the sample, which is performed by a motorized sample mount. In our experiment the spin waves were effectively excited in a wavevector interval $|\mathrm{k}|<1200 \mathrm{~cm}^{-1}$ (with the upper bound imposed by the width of the antenna). Thus, we investigate the light scattered in forward direction to achieve a high sensitivity in this low-wavevector regime.

Temporal resolution is added by using a time correlated single photon counting method similar to time-of-flight measurements in, e.g., mass spectroscopy. The complete setup is shown in Fig. 3. As the frequency selecting device we use a tandem Fabry-Perot interferometer in multipass configuration (for a detailed description see [10]). A pulse generator generates pulses of typically 10-30 ns duration with a repetition rate of $1 \mathrm{MHz}$. The pulse is sent to a microwave switching device to create a pulsed microwave field and to generate a spin wave pulse. The output signal from the pulse generator is also used to start a 24 bit reference counter counting the output pulses of a $1.2 \mathrm{GHz}$ time base. If the spin wave pulse crosses the laser spot, light is inelastically scattered, and the output signal of the photon detector is used to stop the reference counter. The counter content is now a measure of the elapsed time between the launch of the spin wave pulse and the arrival at the position of the laser spot. A memory cell of a memory array addressed by the content of the counter is incremented by one and the procedure is repeated. After accumulating a large number of events the content of the memory array represents the temporal variation of the spin wave intensity at the current position of the laser spot. By repeating the procedure for different positions of the laser spot on the sample, a two-dimensional map of the spin wave intensity is constructed for each value of delay time. We arrange the data in a digital video animation with

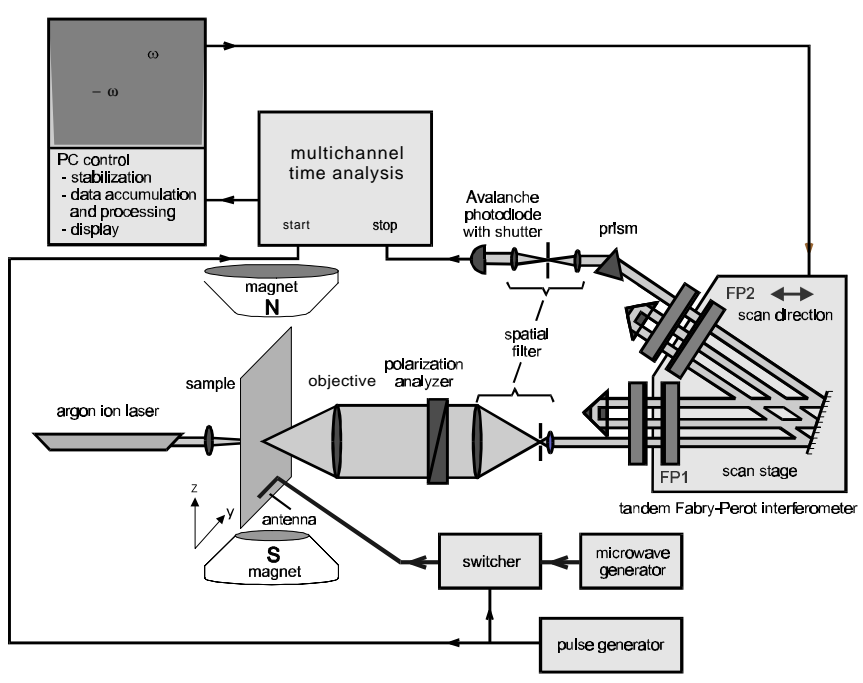

Fig. 3: Schematic layout of the Brillouin light scattering apparatus with space and time resolution. For a discussion of the components see the main 
each frame representing the spatial distribution of the spin wave intensity for a given delay time [28]. Thus, the entire system is a digital signal processing device which interacts with a PC via a RS232 interface. The device can handle up to $2.5 \cdot 10^{6}$ events per second continuously. A lower limit of about 2 ns on the time resolution is imposed by the intrinsic time resolution of the etalons and the multipass arrangement in the BLS spectrometer. Typical accumulation times are 5 seconds per position of the laser spot. A complete measurement of a two-dimensional spin wave intensity pattern in a YIG film with a sampling area of $2 \times 6 \mathrm{~mm}^{2}$ and a mesh size of $0.1 \mathrm{~mm}$ takes a little more than two hours including dead times caused by sample positioning.

\section{Experimental results}

The samples were mounted on a standard microstriptransducer structure with an input antenna of width $50 \mu \mathrm{m}$ and length $2.5 \mathrm{~mm}$. The antenna is connected to a high speed switcher for the pulsed measurements which allows microwave pulses of a time duration $t \geq 10 \mathrm{~ns}$. The switcher in turn is connected to a generator / network analyzer / amplifier unit, which provides a microwave input power of up to $1 \mathrm{~W}$.

The experiments were performed on two different types of ferrimagnetic garnet films: Yttrium Iron Garnet $\left(\mathrm{Y}_{3} \mathrm{Fe}_{5} \mathrm{O}_{12}\right.$, YIG) and Bismuth substituted Iron Garnet $\left(\mathrm{Lu}_{0.96} \mathrm{Bi}_{2.04} \mathrm{Fe}_{5} \mathrm{O}_{12}, \mathrm{BIG}\right)$ films. All samples were epitaxially grown onto (111)-oriented gadolinium-gallium garnet substrates. All BIG films have a thickness of $L=1.5 \mu \mathrm{m}$ whereas the YIG films have a thickness of 5-7 $\mu \mathrm{m}$. Although the dissipation parameter $\omega_{\mathrm{r}}$ is larger in BIG, this material generates a much higher BLS signal due to its higher magneto-optical activity at the $514 \mathrm{~nm}$ laser line. Thin garnet films show a higher spatial attenuation for spin waves due to the lower group velocity of spin waves in these films. The spatial attenuation parameter $\kappa^{\prime \prime}$ is given by $\kappa^{\prime \prime}=$ $\omega_{\mathrm{r}} / v_{\mathrm{g}}$. Since $v_{\mathrm{g}} \propto L$ (see Eqs. (1-4)), we find $\kappa^{\prime \prime} \propto 1 / L$. Although the attenuation might seem to be significant, the high dynamic range of our setup allows us to observe the propagation of spin waves over a large enough distance in the range of up to $10 \mathrm{~mm}$. For the presentation of the data obtained in thin BIG-films with large spatial attenuation we use a contrast enhancement procedure: We find, that, averaged over the $y$-axis, i.e., perpendicular to the mean direction of propagation, the decay in intensity as a function of the distance from the antenna is exponential, and it is given by $\exp \left(-2 \kappa^{\prime \prime} z\right)$ with $z$ the distance from the antenna. By determining $\kappa^{\prime \prime}$ and multiplying the original data by $\exp \left(2 \kappa^{\prime \prime} z\right)$ we transform the data into a representation with compensated attenuation. The procedure is described in detail in [27]. In thicker YIG films this procedure is not necessary.

\subsection{Reciprocity and non-reciprocity of bulk and sur- face waves}

It is well known, that magnetostatic surface waves (MSSW) exhibit a non-reciprocal spectrum and non-reciprocal propagation properties [15]. The non-reciprocal origin of surface waves manifests itself in an asymmetrical excitation of these waves by microwave antennas. This leads to a high, up to $10 \mathrm{~dB}$, asymmetry in the excitation efficiency between two opposite directions of the wavevectors perpendicular to the antenna aperture [29]. The excitation asymmetry is inverted, if the applied magnetic field is reversed.

Contrary to that, the volume waves, i.e., BVMSW modes propagating along the direction of the bias magnetic field, are completely reciprocal. Therefore, these modes show a symmetrical pattern of excitation and propagation along the both in plane directions normal to the antenna.

These very fundamental properties are easily visualized using space resolving Brillouin light scattering. Measurements of intensities of spin waves excited by a microstrip antenna for different orientations of the bias magnetic field were performed in a YIG film (thickness $7 \mu \mathrm{m}$, width $2 \mathrm{~mm}$, length $10 \mathrm{~mm}$ ). The results of the experiments are shown in Fig. 4 where the spin wave intensity distribution is displayed. Figs. $4 \mathrm{a}, \mathrm{b}$ demonstrate the excitation and propagation of MSSW modes. Here the field direction as shown on the left side of the panels is aligned parallel to the antenna. The position of the antenna is marked by the black bar in each panel. The working frequency is $f_{0}=8446 \mathrm{MHz}$, the field is $H=2238 \mathrm{Oe}$, resulting in a spin-wave wavevector of $k_{0}=76 \mathrm{~cm}^{-1}$; the microwave input power is $P_{\text {in }}=10 \mathrm{~mW}$, ensuring the linear regime of wave propagation. Figures $4 \mathrm{a}$ and $4 \mathrm{~b}$ clearly demonstrate the characteristic asymmetry in the MSSW modes excitation and propagation. As it is also seen from the comparison of Figs. $4 a$ and $4 b$, the propagation

a)
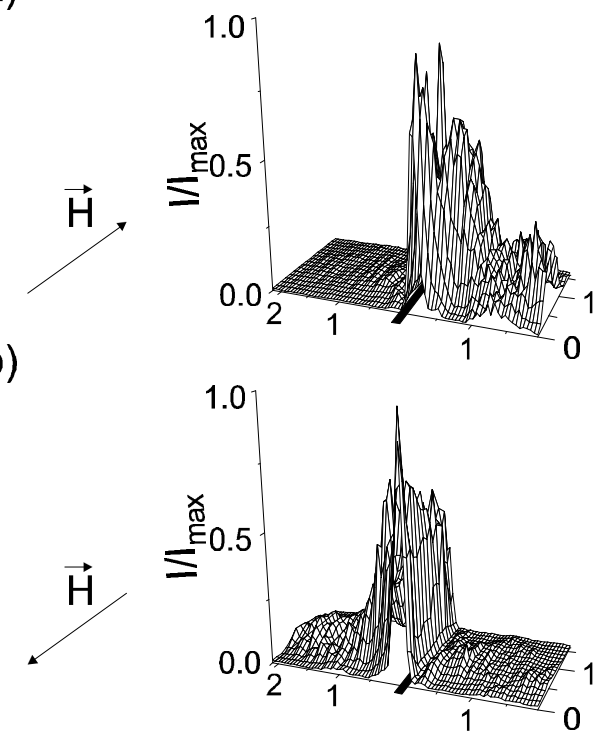

c)

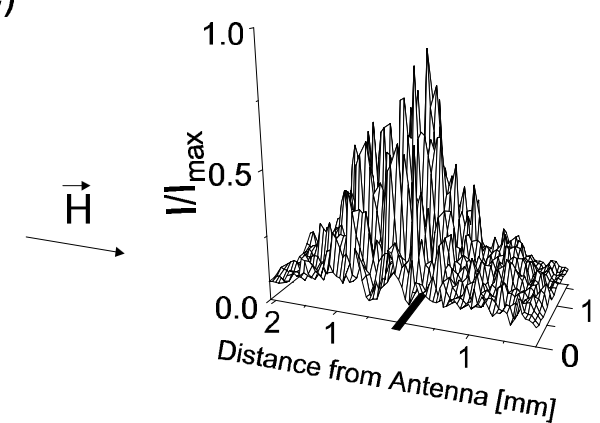

Fig. 4: Three-dimensional plots of the spin wave intensity distribution in a narrow $(\mathrm{w}=2 \mathrm{~mm})$ YIG film sample, when different types of waves are excited by the antenna (shown as a black bar in each frame): (a) and (b) show the excitation of MSSW modes with two opposite orientations of the bias field $\mathrm{H}$; (c) shows the excitation of BVMSW mode. 
direction of the MSSW modes is reversed, if the applied field direction is reversed. Figure $4 \mathrm{c}$ shows the propagation of the BVMSW modes with the field applied perpendicular to the antenna. The working frequency here is $f_{0}=8622 \mathrm{MHz}$, the field is $H=2330 \mathrm{Oe}$, and the carrier wavevector is $k_{0}=88 \mathrm{~cm}^{-1}$. In this case the spin waves are obviously radiated symmetrically in both directions from the antenna as it is expected for reciprocal volume modes.

\subsection{Effect of the antenna aperture}

As we discussed above, the properties of BVMSW modes propagating along the direction of the bias magnetic field depend on the wavevector of the excited spin waves and on the aperture of the antenna. The first experiments on the effect of different wavevectors on the shape of the wave beam propagating from an antenna were performed by Vashkovskii et al [30] using an inductive probe on YIGdiscs. It was found, in agreement with theory, that for low wavevectors two symmetrical beams are radiated at the angles determined by the aperture of the antenna, whereas for the higher wavevectors the spin-wave beam is radiated perpendicular to the direction of the antenna.

These effects are easily demonstrated by space-resolving Brillouin light scattering. For our experiments we used a thin BIG film (thickness $1.5 \mu \mathrm{m}$, width $8 \mathrm{~mm}$, length $10 \mathrm{~mm}$ ) which has a high enough spatial attenuation so that there is no significant mode energy reflected from the film edges parallel to the antenna that could cause interference patterns. The static magnetic field is $H=2090 \mathrm{Oe}$ and the input power is $P_{i n}=10 \mathrm{~mW}$ which guarantees the linear regime of spin wave propagation. The measured two-dimensional distributions of the spin wave intensities for two different cases are displayed in Fig. 5. The intensities are displayed in a gray scale with white/black indicating high/low intensities. The attenuation is corrected as described above to show clearly the details of the spin wave distribution. The position of the input antenna is marked by the dashed bar on the left. Figure 5a corresponds to a small $\left(k_{0 z}=44 \mathrm{~cm}^{-1}\right)$ and Fig. $5 \mathrm{~b}$ to a large $\left(k_{0_{z}}=300 \mathrm{~cm}^{-1}\right)$ value of the carrier wavevector. For low values of $k_{0 z}$ the spin waves are radiated in a large angu- a)

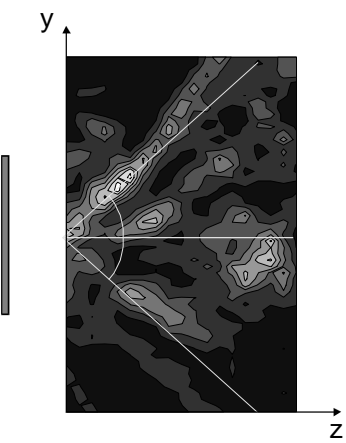

b)

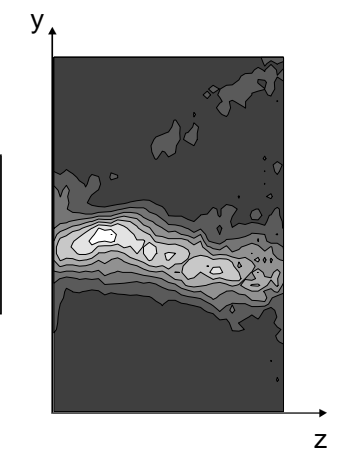

Fig. 5: Two-dimensional plots of spin wave intensity distribution in a large BIG sample for two different values of the carrier wavevector $\mathrm{kOz}$ of the excited BVMSW: (a) shows the case of relatively small carrier wavevector $(\mathrm{k} 0 \mathrm{z}=44 \mathrm{~cm}-1 \sim 2$ /la). Here the waves are exited in a large angle range and two preferential directions of wave group velocity corresponding to $\pm \mathrm{m}$ are clearly seen. Theoretical values of $\mathrm{m}= \pm 42^{\circ}$ are shown by white lines; (b) shows the case of relatively large carrier wavevector $(\mathrm{k} 0 \mathrm{z}=300 \mathrm{~cm}-1>2$ /la). In this case waves are mostly radiated perpendicular to the antenna aperture. lar range, and two preferential directions of the wave beam propagation are clearly visible. They agree very well with the theoretically calculated values of the angle $\theta_{m} \approx 43^{\circ}$ (see Fig. 2) for the conditions of our experiment. These theoretically calculated directions are shown by white lines. For a large wavevector value the beam is mostly radiated in the direction perpendicular to the antenna (Fig. 5b). These results are in good agreement with theoretical results that were discussed in the introduction and with the experimental results described in [30].

The performed experiments also demonstrate the influence of the size of the antenna aperture on the direction of the propagation profile of excited spin waves. The antenna used for the measurements has a length of $2.5 \mathrm{~mm}$, and for sufficiently large carrier wavevectors $k_{0 z}>k_{y} \max$ it creates a spin wave beam in a small angle perpendicular to the antenna, as it was shown above. This is illustrated in more detail in Fig. 6 where the intensity distribution of linearly excited BVMSW modes in a large YIG-Film (thickness $7 \mu \mathrm{m}$, width $18 \mathrm{~mm}$, length $26 \mathrm{~mm}$ ) is shown. The static magnetic field is $H=2109 \mathrm{Oe}$, the working frequency is $f_{0}=7980 \mathrm{MHz}$, and the calculated value of the carrier wavevector is $k_{0 z}=80 \mathrm{~cm}^{-}$ 1. The position of the antenna is shown by the dashed bar on the left. An important effect can be seen in the magnified region of Fig. 6. Here the film has a defect, and the wave is scattered from the defect, which acts as a point-like antenna. The directions of the radiated two sharp shadow beams with the angle between them of $2 \theta_{\mathrm{m}}=85^{\circ}$ are in a good agreement with the theoretical value of the angle $\theta_{\mathrm{m}} \approx 42^{\circ}$ calculated for the conditions of this experiment as described in the introduction (see also Fig. 2).

The above experiments performed on linear waves reproduced in detail well-known properties of dipolar spin waves in tangentially magnetized films and, therefore, confirmed the reliability, sensitivity and resolution of the developed BLS setup. Next stationary and non-stationary nonlinear diffraction processes of spin waves in tangentially magnetized YIG film, and the effect of self-focusing of these waves will be discussed.

\subsection{Nonlinear stationary wave beams}

Stationary self-channeling and initial stages of self-focusing of dipolar spin waves in garnet films were studied earlier by

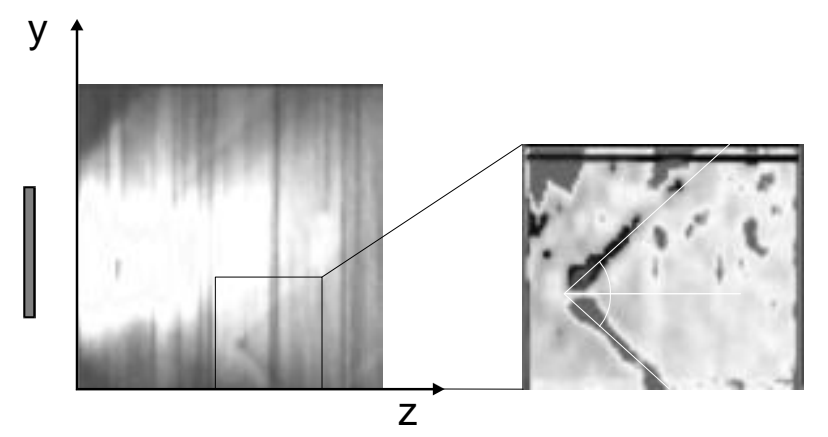

Fig. 6: Two-dimensional spin wave intensity distribution in a large YIG sample. Here dark areas correspond to low and light areas to high spin wave amplitudes. The antenna is shown as a dashed bar on the left side. A part of the propagating wave beam is scattered by a point-like defect which can be seen in the lower right part of the figure. This region in a magnified scale is shown in the inset. White lines show the calculated direction of $\theta_{\mathrm{m}}$ for our experimental condition. 
Boyle et al. [23], who examined the case of spectrally wide beams of both MSSW and BVMSW modes. In their experiments they observed nonlinear beam shaping and some evidence of self-channeling for BVMSW beams, while the MSSW beam (as it is expected from theory) did not demonstrate any significant nonlinear diffraction effects. The evidence of nonlinear self-focusing of dipolar spin wave beams was presented in our previous short paper [27], where spectrally narrow BVMSW beams in a narrow waveguide (width $2 \mathrm{~mm}$ ) were studied. One of the results was the appearance of a snake-like structure in the data which is caused by the interaction of transverse waveguide modes [31]. This structure is caused by the influence of the lateral boundaries of the waveguide. Different width-modes of the waveguide, excited by the microwave antenna, propagate with different phase velocities, and interfere with each other creating a snake-like pattern in the spin wave intensity in the waveguide.

To exclude the effect of boundaries in current measurements of self-focusing, a wide BIG sample (width $18 \mathrm{~mm}$ ) was used. The sample was cut out of the same BIG disc from which the sample in [27] was made. In this experiment the static magnetic field was $H=2090 \mathrm{Oe}$, the carrier frequency was $f_{0}=8450 \mathrm{MHz}$, and the resulting carrier wavevector was $k_{0 z}=300 \mathrm{~cm}^{-1}$. The input power was $P_{i n}=10 \mathrm{~mW}$ in the linear and up to $P_{i n}=600 \mathrm{~mW}$ in the nonlinear case. In Fig. $7 \mathrm{a}, \mathrm{b}$ the experimentally measured spin wave intensity distribution is shown for different values of the input power. The intensity maps are corrected for attenuation, and the data is normalized to the maximum power in each map. The antenna is oriented along the $y$-direction and located on the left at $z=0$. In the linear case, displayed in Fig. 7a, the spin wave amplitude slightly changes due to the effect of the diffraction which causes a beam divergence during propagation, so that the spin wave intensity is distributed over a wider range with increasing distance from the antenna. In the nonlinear case the propagation of the spin waves is very different (Fig. 7b). The beam no longer diverges. It converges to a small diameter while the spin wave amplitude increases and a

a)

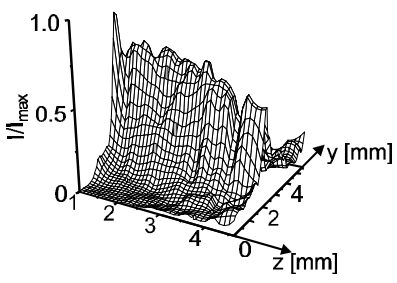

c)

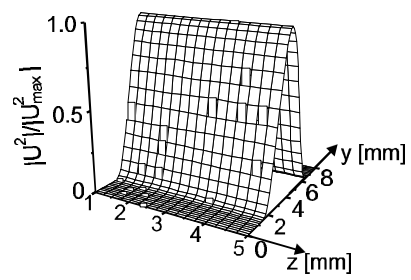

Fig. 7: Stationary self-focusing of BVMSW beams in a large BIG sample:(a) and (b) show experimental distributions of the spin wave normalized intensity in the film plane for the linear $(\mathrm{Pin}=10 \mathrm{~mW}$ ) and nonlinear (Pin $=600 \mathrm{~mW}$ ) regime. A clear self-focusing maximum is seen at the point $(\mathrm{z}=2.5 \mathrm{~mm}, \mathrm{y}=3 \mathrm{~mm})$ in the nonlinear regime (b); (c) and (d) show results of numerical calculations. clear focus of spin wave intensity is observed at the point $z=2 \mathrm{~mm}, y=2 \mathrm{~mm}$. This is the result of the competition between diffraction and nonlinearity, and it can be approximately interpreted as a spatial soliton of a higher order [32]. In the nonlinear regime the formation of a narrow wave channel of constant amplitude parallel to the propagation direction $z$ was not observed in these experiments, probably due to the fact, that the spatial attenuation in the film is large and can play a significant role in the beam shaping process. Since the spin wave beam looses energy due to dissipation during propagation, the beam finally diverges when its amplitude becomes so small, that the diffraction effect dominates, as is the case for $z>2.6 \mathrm{~mm}$ in Fig. 7b.

\subsection{Nonlinear spin wave pulses - spin wave bullets}

In the experiments discussed above only stationary effects of the spin wave propagation were studied. In this case dispersion played no role. If, however, the propagation of short pulses is considered, the dispersion causing the spreading of a wave packet in the propagation direction in the linear case might influence the mode propagation significantly. To measure the real two-dimensional spatio-temporal self focusing, we used a large YIG sample (thickness $7 \mu \mathrm{m}$, width $18 \mathrm{~mm}$, length $26 \mathrm{~mm}$ ) with a FMR linewidth of $2 \Delta H=0.6$ Oe. The static magnetic field was $H=2098 \mathrm{Oe}$, and the carrier frequency was chosen to be $f_{0}=7970 \mathrm{MHz}$ which results in a carrier wavevector $k_{0 z}=50 \mathrm{~cm}^{-1}$. The duration of the microwave input pulse was chosen to be $T=29 \mathrm{~ns}$. The coefficients of the NLS for these parameters are $v_{g}=-4.0 \cdot 10^{6} \mathrm{~cm} / \mathrm{s}, D=1.6 \cdot 10^{3} \mathrm{~cm}^{2} / \mathrm{s}, S=1.1 \cdot 10^{5} \mathrm{~cm}^{2} / \mathrm{s}$, $N=-1.1 \cdot 10^{10} \mathrm{~s}^{-1}$, and $\omega_{\mathrm{r}}=\gamma \Delta H=5.2 \cdot 10^{6} \mathrm{~s}^{-1}$.

Figures 8a,b show the distribution of the spin wave intensities for the above parameters and for two different input powers: $P_{i n}=10 \mathrm{~mW}$ in the linear (Fig. 8a) and $P_{\text {in }}=460 \mathrm{~mW}$ in the nonlinear regime (Fig. 8b). The upper part of each panel shows the spin wave intensity in a threedimensional plot for five different propagation times as indicated in the figure. The data are normalized to the maximum power in each graph. The lower parts shows the crosssections of the wave packets at half-maximum power. In the linear case, the diffraction and the dispersion cause the broadening of the initial wave packet perpendicular to and along the propagation direction, while the amplitude decreases due to dissipation. Here diffraction is considerably larger than dispersion, so that the broadening of the wave packet in the transverse direction is much more pronounced than in the direction of propagation. 
The behavior of the wave packets in the nonlinear case is quite different (Figs. 8c,d). Here the initial high-amplitude wave packet starts to converge, and its amplitude is increasing. Theory (see e.g., [33]) predicts that in the twodimensional case a stable equilibrium between dispersion, diffraction, and nonlinearity is not possible, and nonlinear self-focusing of the wave packet with high enough initial energy should lead to a wave collapse, i.e., all the energy of the packet will be concentrated at one spatial point. In a real medium with dissipation collapse is, of cause, avoided, as the wave packet looses energy. Therefore, in a certain interval of propagation distances nonlinear collapse is stabilized by dissipation, and a quasi-stable strongly localized twodimensional wave packet, a spin wave bullet, is formed. The existence of stable two- and three-dimensional wave packets in a focusing media, where collapse is stabilized by saturation of nonlinearity, has been predicted for optical wave packets in [14]. This effect has not been observed experimentally in optics so far, probably, due to the fact that in optical fibers, where most of the optical experiments are performed, dispersion is several orders of magnitude smaller than diffraction, and it is not possible to observe the influence of both effects on a propagating wave packet simultaneously. In a YIG film diffraction is still substantially larger than dispersion, but the difference is much smaller than in optical fibers. Therefore, here it is possible to observe real spatio-temporal two-dimensional self-focusing of the propagating BVMSW packet.

It is clear from Fig. 8b, that the point of a spatio-temporal a)

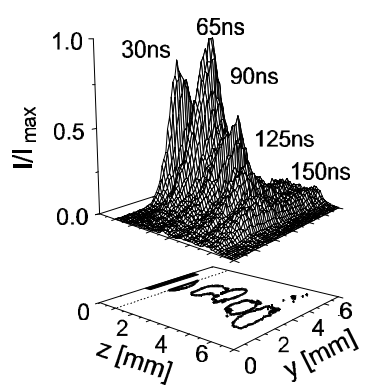

c)

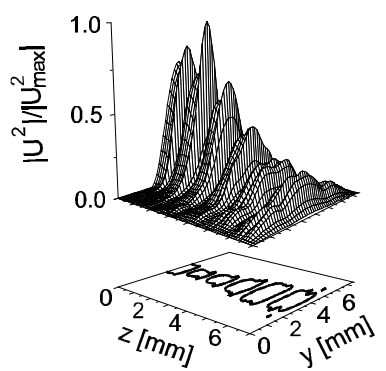

b)

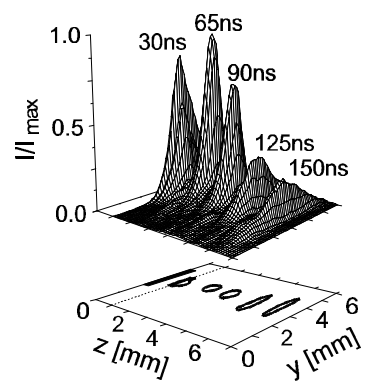

d)

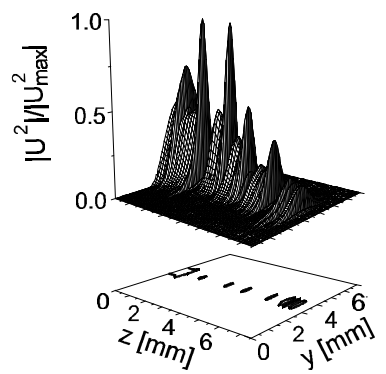

Fig. 8: Non-stationary self-focusing of a two-dimensional BVMSW packet. The upper parts of the pictures show the spin wave intensity distribution in the film created by the propagating pulse of the duration $\mathrm{T}=29 \mathrm{~ns}$ at five successive moments after the moment of a pulse launch from the antenna as indicated in the figure. The lower parts of the panels each show the crosssections of the propagating pulse at half-maximum power. Panels (a) and (b) show experimental data for linear $(\mathrm{Pin}=10 \mathrm{~mW})$ and nonlinear (Pin $=460 \mathrm{~mW}$ ) regimes. Spatio-temporal self focusing is clearly seen for $\mathrm{T}=65 \mathrm{~ns}$ in the nonlinear regime. Panels (c) and (d) show the results of numerical calculations for corresponding parameters. focus (i.e., the point where the amplitude of the packet has a maximum) is located near $z=2-2.5 \mathrm{~mm}(50-60 \mathrm{~ns})$. The transverse width of the packet (along the axis $y$ ) at this point has a minimum. Behind this point the packet moves for some time without a significant change in its shape $(t=50$ $100 \mathrm{~ns}$ ), only its amplitude decays due to dissipation. This is the region of existence of the quasi-stable spin wave bullet. Finally the energy in the wave packet is so small, that the influence of the nonlinearity vanishes and the packet diverges as in the linear case $(t>110 \mathrm{~ns})$.

This effect is further illustrated in Figs. 9a,b, where the width of the packet perpendicular to $\left(L_{y}\right)$ and along the $\left(L_{z}\right)$ propagation direction, and the peak-amplitude of the wave packets are shown as functions of the propagation time in the linear (Fig. 9a) and nonlinear (Fig. 9b) case. For $25 \mathrm{~ns}<t<40 \mathrm{~ns}$ the packet enters the range of measurement. In the linear case (Fig. 9a) the packet behaves monotonously, and the width of the packet is continuously increasing while the amplitude is decreasing. Fig $9 \mathrm{~b}$ shows the nonlinear regime where the packets evolution is different. After the packet entered the measurement region $L_{y}$ and $L_{z}$ rapidly decrease

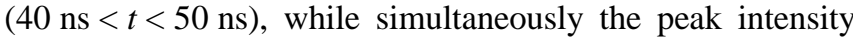
$P_{\text {peak }}$ increases with a minimum in width and a maximum in
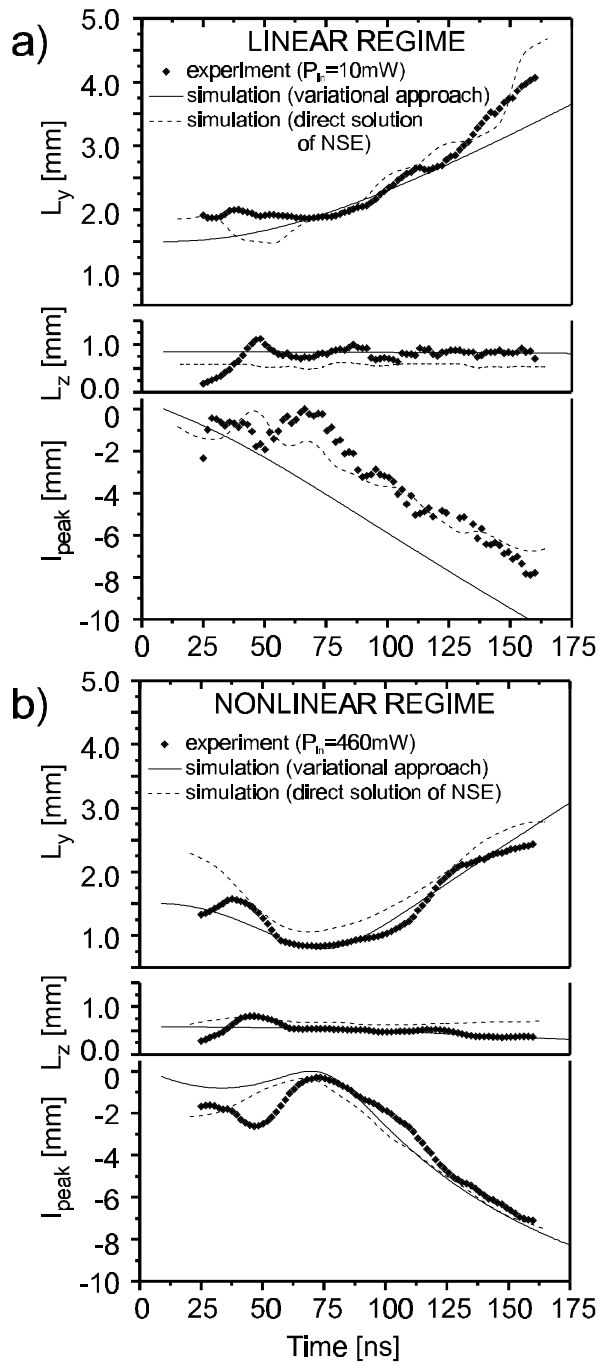

Fig. 9: Time evolution of the transversal (Ly) and the longitudinal (Lz) width of the spin wave packet and the packets amplitude: (a) shows the linear and (b) shows the nonlinear regime. The symbols show the result of the experiment, the straight line shows the result of a simulation based on a variational approach and the dashed line shows the result of a simulation based on the numerical solution of the nonlinear Schrödinger equation. 
intensity at $t=70 \mathrm{~ns}$, where a spin wave bullet is formed. For $t>70 \mathrm{~ns}$ the amplitude of the bullet decays due to the dissipation while $L_{y}$ and $L_{z}$ stay constant. At $t>100 \mathrm{~ns}$ the amplitude is so small that the spatio-temporal focusing effect vanishes and the bullet starts to broaden.

Figure 10 shows the evolution of the spin wave bullet sizes with increasing input power. It is clear that both in-plane sizes of the bullet decrease with increasing power and become closer to each other. This is a typical behavior of a two-dimensional wave packet approaching the collapse point (see e.g. [33]).

\section{Numerical modeling of nonlinear spin wave dif- fraction and propagation}

\subsection{Direct numerical simulation using the $(2+1)$ - dimensional nonlinear Schrödinger equation}

We model the dynamics of nonlinear spin waves in YIG films numerically using the model of a $(2+1)$-dimensional nonlinear Schrödinger equation (NSE) written in the laboratory frame Eq. (9). We use the "magnetic wall" boundary conditions at the lateral edges of the film: $U\left(z, t ; y=0, Y_{0}\right)=0$. The boundary value of the dimensionless spin wave amplitude $U$ at the input antenna is assumed to have the form: $U(z=0, t, y)=F(t) \Phi(y)$, where $\Phi(y)$ is the distribution of the boundary amplitude in the transverse direction, and $F(t)$ is the temporal dependence of the input spin wave amplitude. In the case of two-dimensional modeling $F(t)$ is chosen to be a rectangular pulse of duration $T$, while in the case of a stationary nonlinear propagation, $F(t)$ is assumed to be constant. The initial transverse distribution of the signal is assumed to be cosinusoidal $\Phi(y)=U_{0} \cos [\pi(2 y-W) / 2 W]$, with the width at base equal to $W$. The initial condition for the two-dimensional modeling is the absence of wave perturbations in the propagation region.

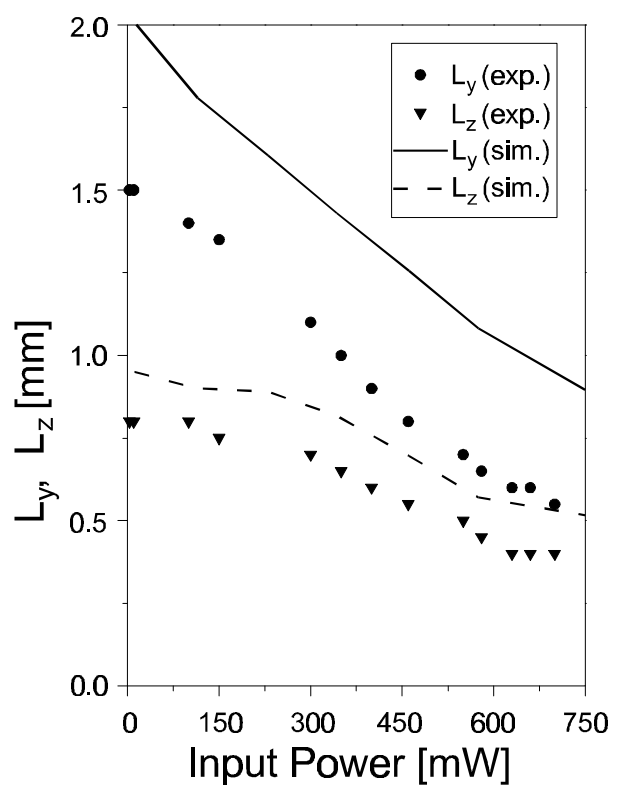

Fig. 10: Widths Ly and Lz of the dipolar spin wave packet of halfmaximum power measured at the focal point (point of the maximum peak power) shown as functions of the input power Pin: symbols: experiment, lines: numerical simulation based on the solution of the nonlinear Schrödinger equation.
When solving the stationary nonlinear diffraction problem, we are calculating the dependence $|U(z, y)|^{2}$ for $z>0$ when the value $U(z=0, y)$ is given at the boundary. When solving the non-stationary problem of a two-dimensional pulse diffraction, it is necessary to obtain $|U(z, t, y)|^{2}$ for all propagation times.

The most popular method for solving the NSE Eq. (9) with homogeneous boundary conditions is the method of splitting by physical factors within the framework of a fast Fourier transform (FFT) [34]. In our case, however, the boundary conditions at $z=0$ are not homogeneous. Therefore, unlike the case of nonlinear optics, the direct use of FFT is not convenient, and the finite difference methods are used.

In the one-dimensional case of stationary wave diffraction, the two-layer Crank-Nicolson implicit numerical scheme is used. To investigate non-stationary two-dimensional wave propagation and diffraction, the alternative directions (ADI) (or Peaceman - Rachford) method is realized [35]. In this method, the first half-step in the calculation uses an approximation at the upper temporal layer along the $z$ direction, while the second half-step uses the analogous approximation along the $y$-direction. The numerical realization of such a numerical scheme is made by a sweep method along the $z$ and $y$-direction for each fractional step. Since the nonlinear term must be evaluated at an unknown temporal step, and in order to achieve a better approximation, we use several iterations, which converge very quickly. Three iterations are sufficient for a full convergence in most cases. The control of accuracy of the calculations is made by means of checking the quadratic conservation integral of Eq. (9), and by subdividing the temporary and the spatial steps of the difference scheme. Also, other methods of numerical simulations like splitting by physical factors are used to check the results of simulations, and a good agreement with the ADI method is obtained.

The modeling of stationary wave propagation and diffraction was made for the following parameters of the film: the length of the film is $Y_{0}=10 \mathrm{~mm}$, the width of the film $Z_{0}=8 \mathrm{~mm}$, and the initial width of the input wave packet at base is $W=5 \mathrm{~mm}$. The parameters of the NSE model for the stationary nonlinear diffraction in a BIG film (see Fig. 7) are: diffraction coefficient $S=3.7 \cdot 10^{3} \mathrm{~cm}^{2} / \mathrm{sec}$, and wave dissipation $\omega_{\mathrm{r}}=7.9 \cdot 10^{6} \mathrm{sec}^{-1}$. The wave group velocity is $v_{g}=-$ $8.6 \cdot 10^{5} \mathrm{~cm} / \mathrm{sec}$, while the nonlinear coefficient is $N=-$ $1.2 \cdot 10^{10} \mathrm{sec}^{-1}$. The results of the calculation are presented in Fig. $7 \mathrm{c}$ for the linear regime $\left(U_{0}^{2}=6.2 \cdot 10^{-4}\right.$ or 0.9 of the nonlinearity threshold defined by Eq. (10)), and in Fig. 7d for the nonlinear regime $\left(U_{0}^{2}=2.4 \cdot 10^{-2}\right.$ or 35 times higher than the nonlinearity threshold). The numerical data are corrected for attenuation.

It is clear from Fig. 8, that in the linear regime, both in theory and in experiment, the only observed effect is a weak diffraction of the BVMSW beam. In the nonlinear regime the nonlinear self-focusing of the beam is clearly seen both in the experimental and in numerical data, and the position of the focal point is very well described by the numerical model.

The non-stationary (two-dimensional) modeling of BVMSW pulse propagation and diffraction in a YIG film sample was done for the following parameters: film length $Z_{0}=26 \mathrm{~mm}$, 
film width $Y_{0}=18 \mathrm{~mm}$, and the initial transverse width of the input wave packet $W=5 \mathrm{~mm}$. The NSE coefficients are: $v_{g}=-3.9 \cdot 10^{6} \mathrm{~cm} / \mathrm{sec}, \quad$ dispersion coefficient $D=1.6 \cdot 10^{3} \mathrm{~cm}^{2} / \mathrm{sec}, \quad$ diffraction coefficient $S=1.1 \cdot 10^{5} \mathrm{~cm}^{2} / \mathrm{sec}$, wave dissipation $\omega_{\mathrm{r}}=5.2 \cdot 10^{6} \mathrm{sec}^{-1}$, and nonlinearity coefficient $N=-1.1 \cdot 10^{10} \mathrm{sec}^{-1}$. In this case, the total width of the film is large enough for the input pulse not to reach the boundaries of the film. The duration of the input pulse is chosen to be $T=29 \mathrm{~ns}$, as in the experiment.

The results of this numerical modeling are presented in Fig. $8 \mathrm{c}$ for the linear case $\left(U_{0}^{2}=1.2 \cdot 10^{-4}\right.$ or 0.25 of the nonlinearity threshold defined by the Eq. (10)) and in Fig. 8d for the nonlinear case $\left(U_{0}^{2}=2.8 \cdot 10^{-3}\right.$ or 6 times higher than the nonlinearity threshold). It is clear that the calculated results are qualitatively similar to the experimental results presented in Figs. 8a,b and in Fig. 9. We also calculated the dependence of the focused beam sizes on the initial amplitude of the input wave packet. This dependence is presented in Fig. 10. In close agreement with the experiment both sizes of the focused wave packet are decreasing with the increase of the input amplitude. Also it can be clearly seen that with the increase of the input power both sizes of the wave packet become closer to each other. In other words, an initially elliptic wave packet becomes more and more circular when it is approaching collapse. This feature of the collapsing wave beams was previously noted in nonlinear optics (see e. g. $[33,36])$.

\section{2 Variational Approach}

Another approach to the numerical modeling of the observed non-stationary self-focusing of two-dimensional BVMSW packets is the so-called variational method [37]. In the framework of this method it is possible, to achieve a better qualitative understanding of the process under investigation since semi-analytical results can be obtained by this method. Because our problem involves higher dimensions and dissipation, one common technique to obtain these analytical results is based on the collective-coordinate method which assumes that the main effect of the perturbation is the (generally speaking) adiabatic modulation of the parameters of the problem. The collective-coordinate method is based on the variational approach first applied to one-dimensional solitary waves by Anderson [37], and then extended to higher dimensions (e.g. [38]). Similar, but less rigorous and detailed methods are the ray-optics approach [39] and the moment theory [40]. Here, we develop a rather general approach based on the variational technique that is the very suitable for our problem. The main advantage of our formalism compared to other variants of the variational technique is that it does not require to employ any specific ansatz for the wave packet shape, treating the dynamics in an universal form. Thus, all the coefficients of the model can be easily calculated for any initial profiles of the wave packet using the general expressions Eqs. (15) and (18).

We consider Eq. (9) in the reference frame moving with the group velocity $v_{\mathrm{g}}$ and present it in the variational form $\delta L=\delta[L d t=0$, where

$$
\begin{aligned}
\mathrm{L}=\mathrm{e}^{\mathrm{i} \omega_{\mathrm{r}} \mathrm{t}} \int_{-\infty}^{\infty}\left[\frac{\mathrm{i}}{2}\left(\mathrm{UU}_{\mathrm{t}}^{*}-\mathrm{U}^{*} \mathrm{U}_{\mathrm{t}}\right)+\frac{1}{2} \mathrm{D}\left|\mathrm{U}_{\mathrm{z}}\right|^{2}+\mathrm{S}\left|\mathrm{U}_{\mathrm{y}}\right|^{2},\right. \\
\left.-\frac{1}{2}|\mathrm{~N} \| \mathrm{U}|^{4}\right] \mathrm{dzdy}
\end{aligned}
$$

where we have assumed that the dispersion and diffraction coefficients are positive $(D, S>0)$ and the nonlinear coefficient is negative $(N<0)$, which corresponds to the situation of self-focusing along both in-plane directions. Following the basic strategy of a variational technique, we seek the general solution of the form [41]:

$$
\mathrm{U}(\mathrm{t}, \mathrm{z}, \mathrm{y})=\mathrm{A}(\mathrm{t}) \mathrm{f}_{1}\left(\frac{\mathrm{z}}{\mathrm{w}_{1}}\right) \mathrm{f}_{2}\left(\frac{\mathrm{y}}{\mathrm{w}_{2}}\right) \mathrm{e}^{\mathrm{i} \alpha_{1} \mathrm{z}^{2}+\mathrm{i} \alpha_{2} \mathrm{y}^{2}} \mathrm{e}^{\mathrm{i} \beta}
$$

where the parameters $A, w_{\mathrm{j}}, \alpha_{\mathrm{j}}$ and $\beta$ are slowly varying functions of time. The important feature of our analysis is the fact that the functions $f_{\mathrm{i}}$ are not specified a priori, allowing generally different shapes of the pulse and much better agreement with direct simulations and experimental data. To obtain the system of equations for the slowly varying parameters, we substitute the solution in the form Eq. (12) into the effective Lagrangian Eq. (11) and integrate over the transverse variables $y$ and $z$. Then, the equation of motion can be found as the Lagrangean equation with the averaged Lagrangian $\bar{L}$ as allows:

$$
\frac{\mathrm{d}}{\mathrm{dt}} \frac{\partial \overline{\mathrm{L}}}{\partial \dot{\xi}_{\mathrm{j}}}=\frac{\partial \overline{\mathrm{L}}}{\partial \xi_{\mathrm{j}}}
$$

where $\xi_{\mathrm{j}}=\left\{A, w_{1}, w_{2}, \alpha_{1}, \alpha_{2}, \beta\right\}$ is the whole set of variables. First of all, the first equation for the parameter $\beta$ can be written in a simple form

$$
\frac{\mathrm{dP}}{\mathrm{dt}}=-2 \omega_{\mathrm{r}} \mathrm{P}
$$

i.e., $P=P(0) \mathrm{e}^{-2 \omega_{\mathrm{r}} \mathrm{t}}$,

where

the value

$\mathrm{P}=\mathrm{A}^{2} \mathrm{~W}_{1} \mathrm{~W}_{2} \mathrm{c}_{01} \mathrm{~d}_{01}$ stands for the total power of the spatiotemporal structure, and the numerical parameters are defined by the expression

$$
\left(c_{n m}, d_{n m}\right)=\int_{-\infty}^{\infty} z^{2 n} f_{1,2}^{2 m}(z) d z .
$$

By varying the parameters $\alpha_{1}$ and $\alpha_{2}$ we get

$$
\begin{aligned}
& \frac{\mathrm{d}^{2} \mathrm{w}_{1}^{2}}{\mathrm{dt}^{2}}=4 \mathrm{D} \alpha_{1} \mathrm{w}_{1}^{2}, \\
& \frac{\mathrm{d}^{2} \mathrm{w}_{2}^{2}}{\mathrm{dt}^{2}}=8 \mathrm{~S} \alpha_{2} \mathrm{w}_{2}^{2},
\end{aligned}
$$

which describe the evolution of the beam diameters. These equations should be combined with the equations for $\alpha_{1}$ and $\alpha_{2}$

$$
\begin{aligned}
& \frac{\mathrm{d} \alpha_{1}}{\mathrm{dt}}=\frac{\mathrm{Dc}_{*}}{2 \mathrm{c}_{11} \mathrm{w}_{1}^{4}}-2 \mathrm{D} \alpha_{1}^{2}-\frac{1}{4} \frac{|\mathrm{N}| \mathrm{A}^{2}}{\mathrm{w}_{1}^{2}} \frac{\mathrm{c}_{02} \mathrm{~d}_{02}}{\mathrm{c}_{11} \mathrm{~d}_{01}}, \\
& \frac{\mathrm{d} \alpha_{2}}{\mathrm{dt}}=\frac{\mathrm{Sd}_{*}}{\mathrm{~d}_{11} \mathrm{w}_{2}^{4}}-4 \mathrm{~S} \alpha_{1}^{2}-\frac{1}{4} \frac{|\mathrm{N}| \mathrm{A}^{2}}{\mathrm{w}_{2}^{2}} \frac{\mathrm{c}_{02} \mathrm{~d}_{02}}{\mathrm{c}_{01} \mathrm{~d}_{11}},
\end{aligned}
$$

where

$$
\left(c_{*}, d_{*}\right)=\int_{-\infty}^{\infty}\left(\frac{d f_{1,2}}{d z}\right)^{2} d z .
$$


Combining Eqs. (16) and (17), we finally obtain a closed system of coupled equations for the pulse widths in the form

$$
\left\{\begin{array}{l}
\frac{\mathrm{d}^{2} \mathrm{w}_{1}}{\mathrm{dt}^{2}}=\frac{\mathrm{a}}{\mathrm{w}_{1}^{3}}-\frac{\mathrm{E}}{\mathrm{w}_{1}^{2} \mathrm{w}_{2}} \mathrm{P}(\mathrm{t}) \\
\frac{\mathrm{d}^{2} \mathrm{w}_{2}}{\mathrm{dt}^{2}}=\frac{\mathrm{b}}{\mathrm{w}_{2}^{3}}-\frac{\mathrm{F}}{\mathrm{w}_{1} \mathrm{w}_{2}^{2}} \mathrm{P}(\mathrm{t})
\end{array}\right.
$$

where $P(t)$ is defined by Eq. (14), and the numerical coefficients are:

$$
\begin{aligned}
& \mathrm{a}=\mathrm{D}^{2}\left(\frac{\mathrm{c}_{*}}{\mathrm{c}_{11}}\right), \mathrm{E}=\frac{1}{2}|\mathrm{~N}| \mathrm{D}\left(\frac{\mathrm{c}_{02} \mathrm{~d}_{02}}{\mathrm{~d}_{01}^{2} \mathrm{c}_{01} \mathrm{c}_{11}}\right), \\
& \left.\mathrm{b}=4 \mathrm{~S}^{2}\left(\frac{\mathrm{d}_{*}}{\mathrm{~d}_{11}}\right), \mathrm{F}=\mathrm{S}|\mathrm{N}| \frac{\mathrm{c}_{02} \mathrm{~d}_{02}}{\mathrm{c}_{01}^{2} \mathrm{~d}_{01} \mathrm{~d}_{11}}\right) .
\end{aligned}
$$

System (19) is similar to that derived by means of the rayoptics approach [42], which generally gives wrong results for the coefficients. In the symmetric case, we should impose the conditions $S=D / 2, \alpha_{1}=\alpha_{2}, w_{1}=w_{2}$, and $f_{1}=f_{2}$, so that the system (19) reduces to a single equation for the common width parameter $w$, that can be easily investigated with and without dissipation (see, e.g., the analysis of the similar equation derived by the moment method [43]). In our case, the width parameters are not equal, and the initial wave packet is elliptic, so we need to solve the system (19) numerically.

The results of this solution are compared with the experimental results and the results of direct numerical modeling. They are displayed in Figs. 9a and $9 \mathrm{~b}$ for the linear and nonlinear regimes. We use Gaussian pulse profiles along both in-plane directions and the parameters $v_{g}=-4.0 \cdot 10^{6} \mathrm{~cm} / \mathrm{s}$, $D=1.6 \cdot 10^{3} \mathrm{~cm}^{2} / \mathrm{s}, \quad S=1.1 \cdot 10^{5} \mathrm{~cm}^{2} / \mathrm{s}, \quad N=-1.1 \cdot 10^{10} \mathrm{~s}^{-1}$, $\omega_{\mathrm{r}}=\gamma \Delta H=5.2 \cdot 10^{6} \mathrm{~s}^{-1}, \quad L_{0 y}=1.5 \mathrm{~mm}, \quad L_{0 z}=0.85 \mathrm{~mm}$, and $\mathrm{A}_{0}^{2}=5 \cdot 10^{-2}$. It is clear that the variational approach provides a picture of the initial wave packet evolution that is qualitatively correct, but less accurate than the direct numerical experiment using the NSE model, Eq. (9). It is worth noting, that in order to achieve a qualitative agreement with experiment we had to use in the variational approach a value of the initial packet amplitude that is 20 times larger than in the direct numerical simulation using NSE Eq. (9). This shows that, apart from the deeper physical insight the method provides, the variational method has only limited quantitative applicability in this case.

\section{Conclusions}

In conclusion, a newly developed space- and time-resolved BLS technique was applied for the investigations of linear and non-linear propagation and diffraction of different spin wave modes in garnet films. The non-reciprocal excitation and propagation of magnetostatic surface spin wave as well as the formation of two narrow beams of backward volume magnetostatic waves (BVMSW) from an initially excited broad beam were demonstrated and investigated in detail with high spatial resolution. Both effects, which were separately observed before, are connected with the peculiarities of the linear spectrum of the corresponding mode. The stationary and non-stationary non-linear self focusing of BVMSW was studied. For the first time formation of highly localized quasi-stable two-dimensional packets of spin waves, spin wave bullets, has been observed and investigated. The experimental observations are in a good agreement with results of numerical simulations based on the nonlinear Schrödinger equation and with those of a variational approach.

Support by the Deutsche Forschungsgemeinschaft and National Science Foundation (Grant DMR-9701640) is gratefully acknowledged.

\section{References}

[1] P.E. Wigen (ed.) Nonlinear Phenomena and Chaos in Magnetic Materials (World Scientific, Singapore, 1994).

[2] M.G. Cottam (ed.), Linear and Nonlinear Spin Waves in Magnetic Films and Superlattices, (World Scientific, Singapore 1994).

[3] B.A. Kalinikos, N.G. Kovshikov, A.N. Slavin, Sov. Phys. JETP Lett. 38, 138 (1983).

[4] B.A. Kalinikos, N.G. Kovshikov, A.N. Slavin, Sov. Tech. Phys. Lett. 10, 392 (1984).

[5] P.E. Zilberman, S.A. Nikitov, A.G. Temiryazev, Sov. Phys. JETP Lett. 42, 110 (1985).

[6] N.P. Vlannes, J. Appl. Phys. 61 416, (1987).

[7] A.B. Valyavsky, A.V. Vashkovsky, A.V. Stal'makov, V.A. Tyulyukin, Sov. Phys. Tech. Phys. 34, 616 (1989).

[8] O.V. Geisau, U. Netzelmann, S.M. Rezende, J. Pelzl, IEEE Trans. Mag. 26, 1471 (1990).

[9] A.A. Solomko, Y.A. Gaidai, O.V. Kolokoltsev, Abstracts of ICM-94, p 368, Warsaw, Poland (1994).

[10] B. Hillebrands, Rev. Sci. Instr. 70, 1589 (1999).

[11] O. Büttner, M. Bauer, A. Rueff, S.O. Demokritov, B. Hillebrands, A.N. Slavin, M.P. Kostylev, B.A. Kalinikos, submitted to Ultrasonics.

[12]M. Bauer, O, Büttner, S.O. Demokritov, B. Hillebrands, to be submitted to Rev. Sci. Inst..

[13]M. Bauer, O. Büttner, S.O. Demokritov, B. Hillebrands, V. Grimalsky, Yu. Rapoport, A.N. Slavin, Phys. Rev. Lett. 81, 3769 (1998).

[14] Y. Silberberg, Opt. Lett. 15, 1282 (1990).

[15] R.W. Damon, J. Eshbach, J. Chem. Phys. Solids 19, 308 (1961).

[16] B. A. Kalinikos, A.N. Slavin, J. Phys. C 19, 7013 (1986).

[17] B. Lax, K.J. Button, "Microwave Ferrites and Ferrimagnetics", McGraw-Hill, New York, 1962.

[18] B.A. Kalinikos, N.G. Kovshikov, A.N. Slavin, Sov. Phys. JETP 67, 303 (1988).

[19] G.B. Witham, Linear and Nonlinear Waves (Wiley, New York, 1974).

[20]A.N. Slavin, Phys. Rev. Lett. 77, 4644 (1996).

[21]A.N. Slavin, I.V. Rojdestvenski, IEEE Trans. Mag. 30, 37 (1994).

[22] M.J. Lighthill, J. Inst. Math. Appl. 1, 269 (1965).

[23]J.W. Boyle, S.A. Nikitov, A.D. Boardman, J.G. Booth, K. Booth, Phys. Rev. B 53, 12173 (1996).

[24]M.Chen, M.A. Tsankov, J.M. Nash, and C.E. Patton, Phys. Rev. B 49, 12773 (1994).

[25]M.Chen, M.A. Tsankov, J.M. Nash, and C.E. Patton, Phys. Rev. Lett. 70, 1707 (1993).

[26]A.K. Ganguly, D.C. Webb, IEEE Trans. Microwave Theory and Techniques, MTT-23, 998 (1975).

[27] M. Bauer, C. Mathieu, S.O. Demokritov, B. Hillebrands, P.A. Kolodin, S. Sure, H. Dötsch, V. Grimalsky, Yu. Rapoport, A.N. Slavin, Phys. Rev. B 56, R8483 (1997).

[28] Video sequences in the AVI format can be downloaded from our home page http://www.physik.uni-kl.de/w_hilleb.

[29]P.R. Emtage, J. Appl. Phys. 49, 4475 (1978).

[30]A.V. Vashkovskii, A.V. Stal'makhov, D.G. Shakhnazaryan, Izvestya Vysshikh Uchebnykh Zavedenii, Fizika 11, 67 (1988).

[31] O. Büttner, M. Bauer, C. Mathieu, S.O. Demokritov, B. Hillebrands, P.A. Kolodin, M.P. Kostylev, S. Sure, H. Dötsch, V. Grimalsky, Yu. Rapoport, A.N. Slavin, IEEE Trans. Magn. 34, 1381 (1998).

[32] G.P. Agrawal, 'Nonlinear Fiber Optics', Academic Press (1989), Fig. 5.6 . 
[33]L. Berge, Physics Reports 303, 260 (1998).

[34]A.C. Newell, J.V. Moloney, Nonlinear Optics, Addison-Wesley, Redwood, CA (1992.)

[35]W.H. Press, B.P. Flannery, S.A. Teulkovsky, W.T: Vettering, Numerical Recipes, Cambridge Univ. Press (1992).

[36]C.R. Giuliano, J.H. Marburger, A. Yariv, Appl. Phys. Lett. 21, 58 (1972).

[37]D. Anderson, Phys. Rev. A 27, 3135 (1983).

[38]M. Karlsson, D. Andersson, M. Desaix, M. Lisak, Opt. Lett. 16, 1373 (1992).

[39]S.A. Akhmanov, A.P. Sukhorukov, R.V. Khokhlov, Usp. Fiz. Nauk. 13, 19 (1967).

[40]V.M. Pérez-Garcia, M.A. Porros, L. Vázquez, Phys. Lett. A 202, 176 (1995).

[41]D.Anderson, M. Bonnedal, M.Lisak, J. Plasma Physics 23, 115 (1980).

[42] V.P. Nayyar, V.S. Soni, J. Phys. D: Appl. Phys 12, 239 (1979).

[43]M.V. Goldman, K. Rypdal, and B. Hafizi, Phys. Fluids 23, 945 (1980). 